\title{
Mechanical reliability of fullerene/tin-oxide interfaces in monolithic perovskite/silicon tandem cells
}

Michele De Bastiani, ${ }^{1, *}$ Giovanni Armaroli, ${ }^{2}$ Rawan Jalmood, ${ }^{1}$ Laura Ferlauto,,${ }^{2,4}$ Xiaole Li, ${ }^{3}$ Ran Tao, ${ }^{3}$ George T. Harrison, ${ }^{1}$ Mathan K. Eswaran, ${ }^{1}$ Randi Azmi, ${ }^{1}$ Maxime Babics, ${ }^{1}$ Anand S. Subbiah, ${ }^{1}$ Erkan Aydin, ${ }^{1}$ Thomas G. Allen, ${ }^{1}$ Craig Combe, ${ }^{1}$ Tobias Cramer, ${ }^{2}$ Derya Baran, ${ }^{1}$ Udo Schwingenschlögl, ${ }^{1}$ Gilles Lubineau, ${ }^{3}$ Daniela Cavalcoli, ${ }^{2}$ Stefaan De Wolf1,*

1 KAUST Solar Center (KSC), Physical Sciences and Engineering Division (PSE), King Abdullah University of Science and Technology (KAUST), Thuwal 23955-6900, Kingdom of Saudi Arabia.

2 Department of Physics and Astronomy, University of Bologna, Viale Berti Pichat 6/2, 40127, Bologna, Italy.

3 King Abdullah University of Science and Technology (KAUST), Physical Science and Engineering Division, Mechanics of Composites for Energy and Mobility Lab., Thuwal 23955-6900, Saudi Arabia.

4 Interdepartmental Center for Industrial Research of the University of Bologna (CIRI-MAM), Viale Risorgimento 2, 40136, Bologna, Italy

*email: Michele.debastiani@kaust.edu.sa; Stefaan.dewolf@kaust.edu.sa

\section{Supporting Information}

\section{Experimental part:}

Perovskite/silicon tandem fabrication: 4-inches double-sided polished wafers (float-zone, TOPSIL, ndoped, resistivity 1-5 $\Omega \mathrm{cm}^{-1}$ and thickness $250-280 \mu \mathrm{m}$ ) were used as starting point of the bottom cell fabrication. On the surface of the wafer, the randomly textured pyramids were obtained using alkaline solution, the size of pyramids is controlled by interchanging the temperature, the alkaline concentration and processing time. To further improve the quality of the texturing, additive was added during the process. The texturing process was followed by a cleaning step RCA procedure. After the wet processing, the surfaces of the wafers were passivated by the deposition of intrinsic $(i)$ amorphous silicon (a-Si:H) followed by amorphous and nanocrystalline doped layers ( $p$ and $n c-n$ with thickness 12 and 40nm, respectively) deposited in the the Octopus 2 cluster (Indeotec). To create the contacts, ITO and Ag were sputtered on the rear side $(110 \mathrm{~nm}$ and $250 \mathrm{~nm}$, respectively). For the recombination junction at the front side, $20 \mathrm{~nm}$ of ITO were sputtered in the Octopus 2 cluster, followed by $17 \mathrm{~nm}$ of NiOx sputtered with a Angstrom Evovac tool. The NiOx was then passivated using bromobenzoic acid. The perovskite solution (1.7M, CsI $36.4 \mathrm{mg}$, MABr $44.8 \mathrm{mg}$, FAI $389 \mathrm{mg}, \mathrm{PbI}_{2} 922 \mathrm{mg}$, and $\mathrm{PbBr}_{2} 293 \mathrm{mg}$ ) was deposited via spincoating from a 4:1 ratio of Dimethylformamide Dimethyl sulfoxide (DMF:DMSO, Sigma Aldrich) respectively, then the film was annealed for $10 \mathrm{~min}$ at $100{ }^{\circ} \mathrm{C}$ in nitrogen glove box. On top of perovskite, $20 \mathrm{~nm}$ of $\mathrm{C}_{60}$ was thermally evaporated (Angstrom Evovac) as electron transport layer. The C60 layer was covered by $20 \mathrm{~nm}$ of $\mathrm{SnO}_{2}$ deposited by atomic layer deposition (ALD, Picosun). After that, $75 \mathrm{~nm}$ of IZO were sputtered (Angstrom Evovac) to realize the top transparent contact. Lastly, $110 \mathrm{~nm}$ of $\mathrm{MgF}_{2}$ are thermally evaporated (Angstrom EvoVac) where mentioned as an antireflection film.

Kelvin Probe Force Microscopy: Samples were loaded in a Park System NX10 Atomic Force Microscope enclosed in a Glove-Box filled with dry Argon gas. The delamination process was carried out inside the Glove-Box in order to avoid sample degradation due to environmental moisture or oxygen. The AFM maps were acquired in non-contact mode with a NSC36/CrAu-B tip (Mikromasch, k=2 N/m). Measurements were performed in dark in order to avoid light-induced degradation of the samples. All work function values were obtained via calibration of the tip with a Indium-Tin-Oxide sample of known work function.

Scanning Electron Microscopy, Energy Dispersive X-ray analysis: Scanning electron microscopy (SEM) images were taken at $5 \mathrm{kV}$ accelerating voltage with $30 \mu \mathrm{m}$ beam aperture, using a Zeiss Auriga microscope 
equipped with an in-lens detector. Energy Dispersive X-ray (EDX) analysis was performed on the same tool with $20 \mathrm{kV}$ accelerating voltage.

X-ray Photoelectron Spectroscopy, Ultraviolet Photoelectron Spectroscopy: X-ray photoelectron spectroscopy (XPS) was carried out using an (Omicron) multi-probe ultrahigh vacuum (UHV) chamber operated at $10^{-10} \mathrm{mbar}$ and equipped with a monochromated $\mathrm{AlK} \alpha(1486.6 \mathrm{eV}) \mathrm{X}$-ray source $(15 \mathrm{kV}, 26$ $\mathrm{mA}$ ) and a hemispherical EAC125 Sphera 2 analyser with a channeltron detector. CasaXPS software was used for all peak fitting and analysis.

Peeling test: The work of adhesion was evaluated with adhesion experiment using a Yeonjin TXA texture analyzer and kapton tape. Samples were fixed on glass slides and mounted in a $180^{\circ}$ configuration.

Computational method: Density functional theory is used in the generalized gradient approximation of Perdew, Burke, and Ernzerhof (Vienna ab-initio simulation package; ${ }^{1}$ projector augmented wave method; $450 \mathrm{eV}$ plane wave cut-off). A $4 \times 2 \times 1$ (110) supercell of tetragonal $\mathrm{SnO}_{2}$ with space group $\mathrm{P}_{2} / \mathrm{mnm}$ (optimized primitive cell lattice constants of $a=b=4.83 \AA$ and $c=3.25 \AA$, in agreement with the literature $\left.{ }^{2}\right)$ is built. The supercell is augmented with a vacuum layer of $20 \AA$ thickness in the $c$-direction to achieve a slab model of the energetically favorable SnO-terminated (110) surface. ${ }^{2}$ The total energy is converged to $10^{-5} \mathrm{eV}$ and the maximum atomic force is converged to $0.01 \mathrm{eV} \AA^{-1}$.

a

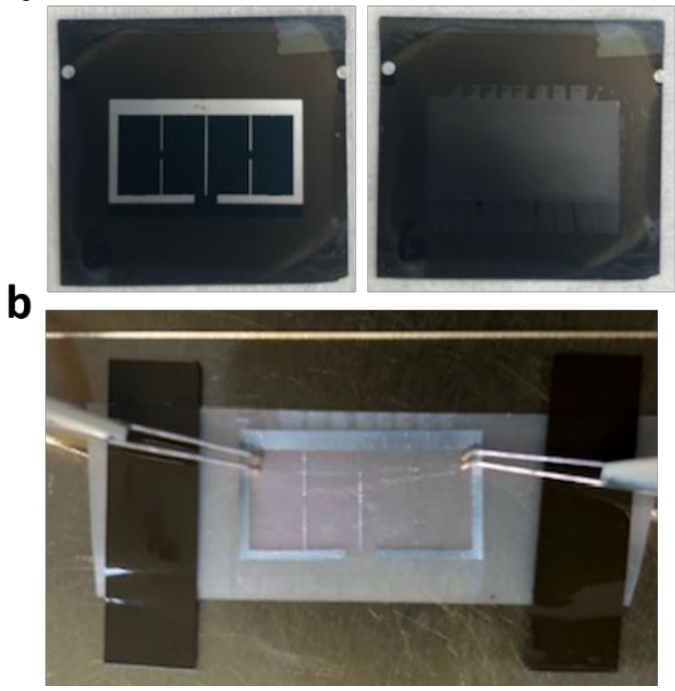

C

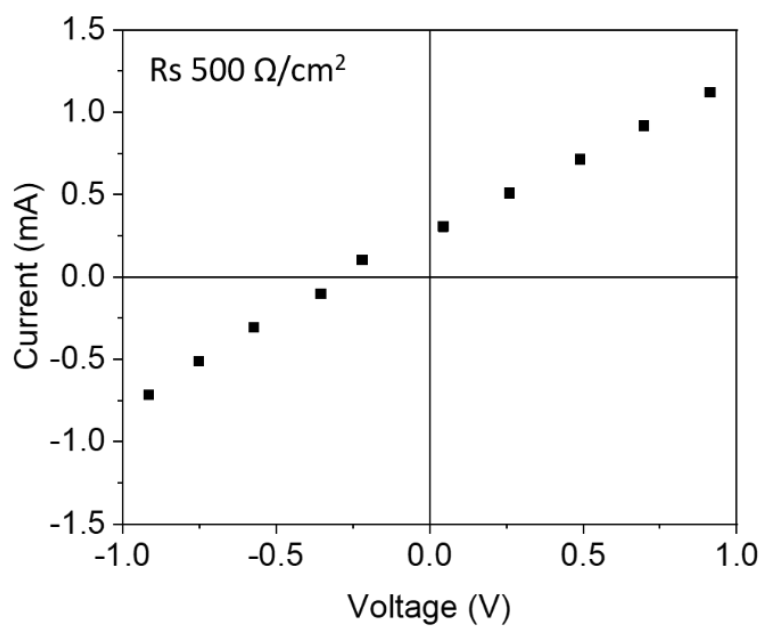

Figure S1. a) Picture of the tandem before and after the peeling of the top electrode. b) Picture of a peeled electrode that highlights the contact setup on the peeling tape, avoiding the silver busbar. c) IV curve with the Rs of the IZO contact probed through the $\mathrm{SnO}_{2}$ layer. 

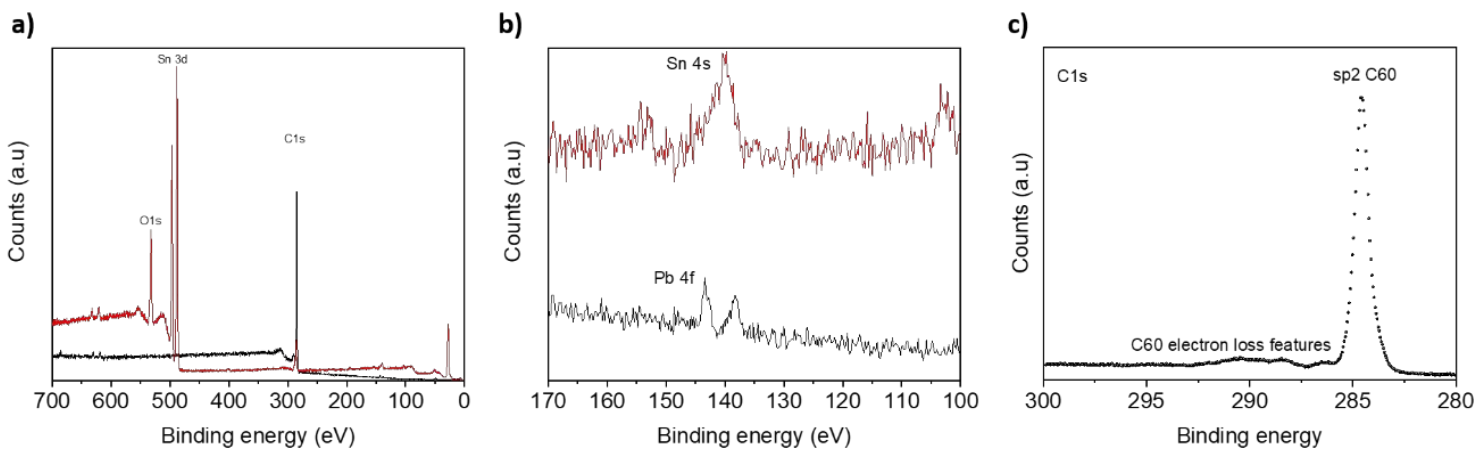

Figure S2. Details of the XPS spectra after the peeling experiment. a) Survey spectra of the emerging surface of the tandem after the peeling of the top contact (black), compared with the peeled surface sticking on the tape (red). b) Magnification of the survey spectrum. c) Details of the C60 features present in the spectra of the emerging surface.
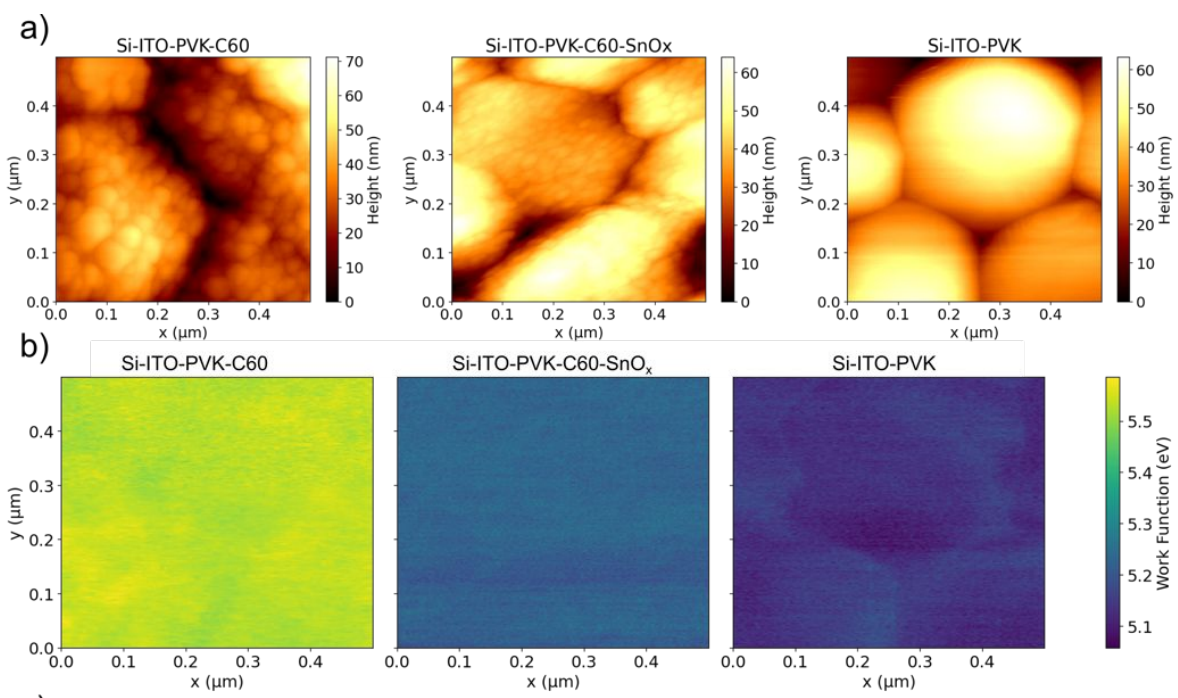

c)
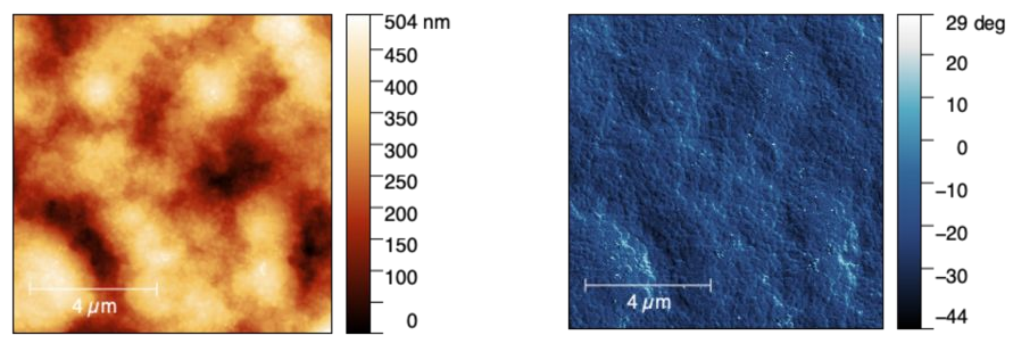

d)
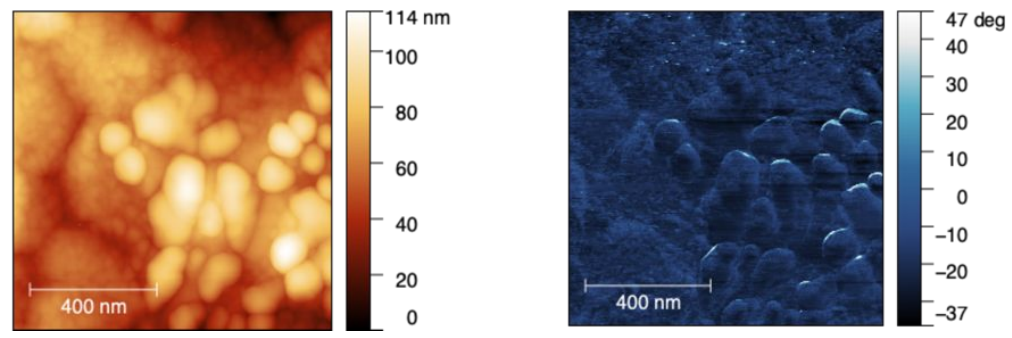
Figure S3. Morphology (a) and KPFM (b) maps of calibration samples. Morphology and Non-ContactMode phase maps of the delaminated solar cell both in $10 \mu \mathrm{m}^{2}(\mathrm{c})$ and $1 \mu \mathrm{m}^{2}(\mathrm{~d})$ scale.

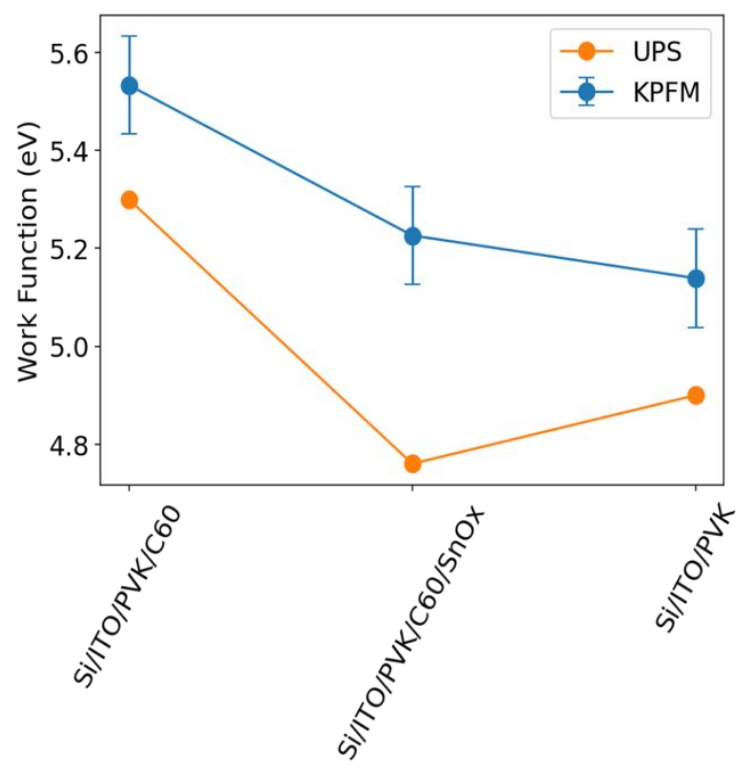

Figure S4. Comparison of Work function values of the reference samples as measured by KPFM and UPS. The lines connecting the dots are meant as a guide for the eye.
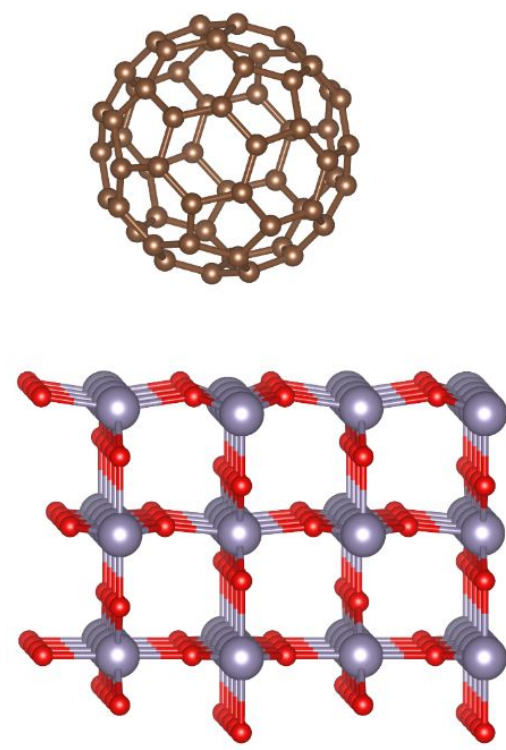

Figure S5. Relaxed structure for the $\mathrm{C}_{60}$ molecule on the SnO-terminated (110) surface of $\mathrm{SnO}_{2}$. Brown, red and grey spheres represent $\mathrm{C}, \mathrm{O}$ and $\mathrm{Sn}$ atoms, respectively. 


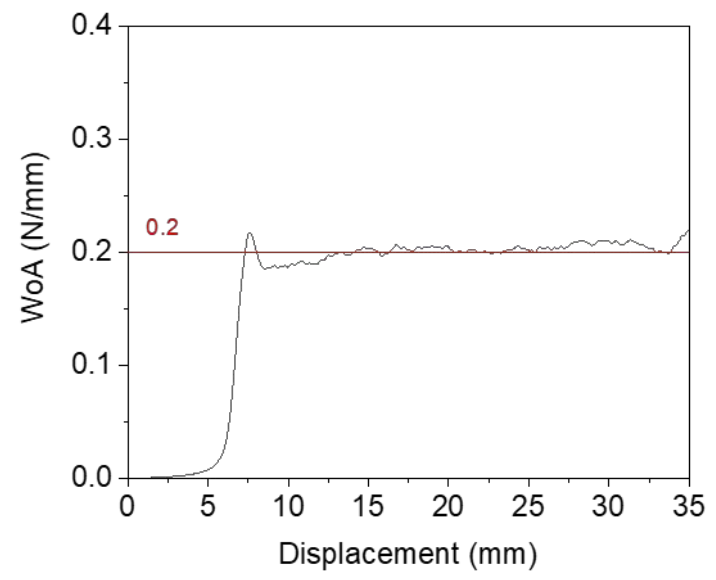

Figure S6. Work of Adhesion of the tape on the textured silicon substrate.

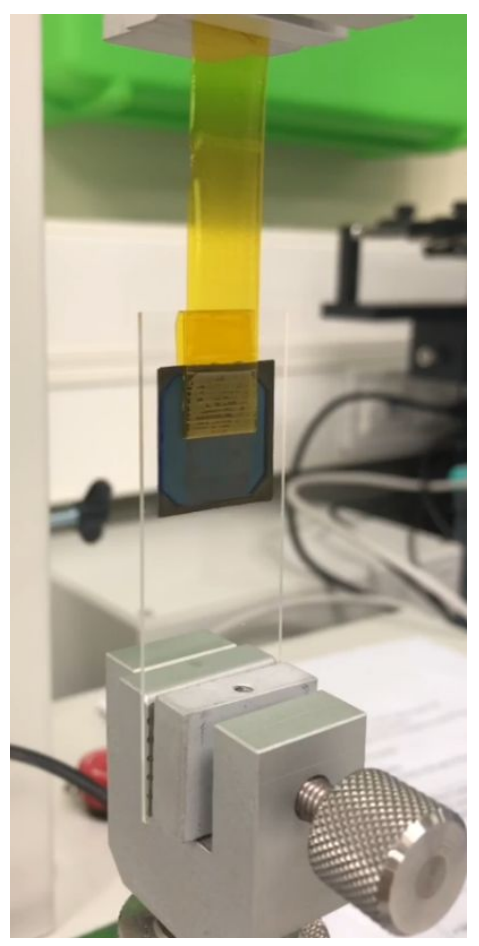

Figure S7. Stick and slip behavior of the delamination (video uploaded).

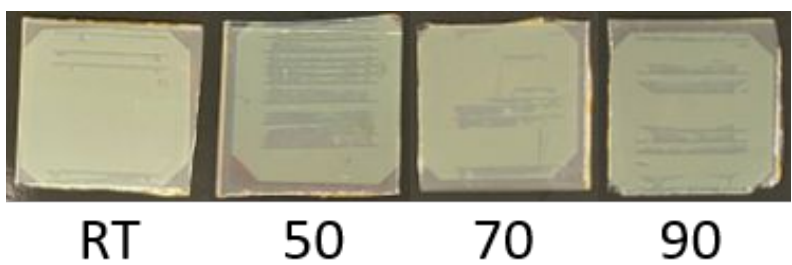


Figure S8. Pictures of the test structures delaminated after different annealing steps. The octahedral shape on the sample is the shadow of the $\mathrm{C}_{60}$ mask during the thermal evaporation. The delamination pattern highlights the stick and slip behavior.

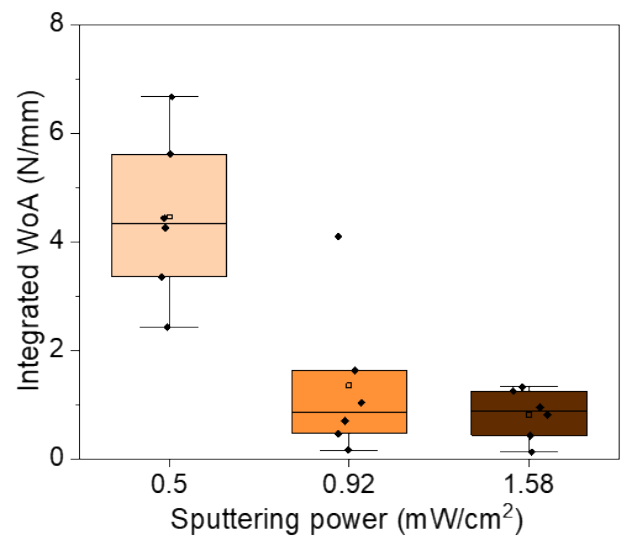

Figure S9. Distribution of the integrated WoA (over the same detached area) for $\mathrm{PVK} / \mathrm{C}_{60} / \mathrm{SnO}_{2} / \mathrm{IZO}$ test structures with IZO deposited at different powers. For each condition, we prepared six samples.

\section{References}

1. Kresse, G.; Joubert, D., From ultrasoft pseudopotentials to the projector augmented-wave method. Physical review $b$ 1999, 59 (3), 1758.

2. Wang, Y.; Mei, X.; Qiu, J.; Zhou, Q.; Jia, D.; Yu, M.; Liu, J.; Zhang, X., Insight into the Interface Engineering of a SnO2/FAPbI3 Perovskite Using Lead Halide as an Interlayer: A First-Principles Study. The Journal of Physical Chemistry Letters 2021, 12, 11330-11338. 